\title{
La adaptación productiva, la mejor respuesta al cambio climático
}

\author{
MSc. Jazmín TAVERA COLONNA
}

La política gubernamental peruana frente al cambio climático se centra en la reducción de las emisiones y mecanismos de desarrollo limpio (MDL), lo que permite prevenir y reducir nuestras emisiones, las que a nivel mundial son proporcionalmente imperceptibles. El 53\% de las empresas manufactureras se encuentran en Lima y el $77 \%$ en la costa, lo que convierte a nuestro sector manufacturero altamente vulnerable al cambio climático.

\section{EL CAMBIO CLIMÁTICO}

De acuerdo a la Organización Meteorológica Mundial OMM o IPCC, el "cambio climático" es un cambio en el valor medio y/o en la variabilidad de las propiedades del clima, cambios de carácter persistente durante un período prolongado, decenios o períodos más largos. Sus causas pueden originarse en dos tipos de factores, cambios naturales del sistema terrestre, o ser consecuencia de la actividad humana. En cambio, la Convención Marco de las Naciones Unidas sobre el Cambio Climático (CMCC), define al cambio climático como un cambio del clima atribuido directa o indirectamente a la actividad humana, la cual altera la composición de la atmósfera a nivel mundial, y suma cambios a la variabilidad climática de origen natural.

De acuerdo a la OMM, de los doce últimos años (1995-2006), once figuran entre los doce más cálidos en los registros instrumentales de la temperatura de la superficie mundial (desde 1850). Este aumento de temperatura está distribuido por todo el planeta, siendo las regiones terrestres más afectas las localizadas cerca a los océanos, acentuándose más en las latitudes septentrionales superiores. El aumento del nivel del mar concuerda con este calentamiento. En promedio, el nivel de los océanos mundiales ha aumentado desde 1961 a un promedio de $1,8 \mathrm{~mm} /$ año, y desde 1993 a 3,1 mm/año, por efecto de la dilatación térmica y el deshielo de los glaciares, y mantos de hielo polares. Datos satelitales desde 1978 indican que la extensión de los hielos marinos árticos ha disminuido en promedio un 2,7 $\%$ por decenio. En Perú el área de mayor impacto se encuentra en Piura. 
De acuerdo con Brooks, N. y Adger N. (2003) del Tyndall Center, el Perú se encontraría entre los diez países más vulnerables ante eventos climáticos junto a países como Honduras, Bangladesh y Venezuela. Esta vulnerabilidad está asociada a la alta dependencia a sectores primarios sensibles al cambio climático, tales como el agrícola y el pesquero, así como al bajo nivel institucional, que dificulta la planificación y ejecución de acciones de adaptación concretas.

El $80 \%$ de nuestra electricidad es generada por centrales hidroeléctricas. En los últimos 30 años se ha perdido el $22 \%$ de la superficie de nuestros glaciares, que son el $71 \%$ de los glaciares tropicales del mundo. Y nuestras instituciones tienen una capacidad de acción limitada. A1 2007 la capacidad actual de nuestros glaciares era de 43 mil millones de m3, siendo el 60\% de nuestro 'stock' de agua, ya que el 40\% restante, 25 mil millones de m3, participa en el flujo hídrico. De acuerdo a las proyecciones que existen sobre cambio climático se proyecta que en esta década el 40\% de nuestro 'stock' de agua en los glaciares se irá en el flujo hídrico. Estos fenómenos tienen un componente antrópico importante, ya que en la década del setenta se dieron en las punas, yungas y quebradas, prácticas de deforestación, sobrepastoreo, ampliación de la frontera agrícola y drenaje de humedales. Prácticas locales que unidas a las emisiones de GEI están revirtiendo totalmente los ecosistemas andinos.

En el 2010, Perú incorpora en la segunda comunicación a la CMNUCC la implementación de proyectos de adaptación al cambio climático. La estrategia se implementó a través del Programa Nacional para el Cambio Climático y la Calidad del Aire (PROCLIM, conformado por 14 instituciones), que formuló prioridades nacionales en base a los estudios de vulnerabilidad climática, que se tradujeron en proyectos en las cuencas de los ríos Piura, Santa y Mantaro.

\section{LA ADAPTACIÓN AL CAMBIO CLIMÁTICO}

La adaptación al cambio climático consiste en el "ajuste en los sistemas naturales o humanos a los estímulos climáticos reales o esperados, o a sus efectos, que modera el daño o aprovecha las oportunidades beneficiosas" (IPCC, 2008). La adaptación es un proceso de adecuación, sostenible y permanente, en respuesta a circunstancias ambientales nuevas y cambiantes que permite reducir la incertidumbre; y que demanda la solución de temas estructurales como presión sobre ecosistemas, exclusión social y pobreza.

En el caso del Perú, los tomadores de decisiones a nivel de políticas de desarrollo son quienes deben internalizar dentro de sus planes de desarrollo el tema de la adaptación al cambio climático: CEPLAN, el MEF y la PCM, los que deben incluir la planificación estratégica para la adaptación en los instrumentos de gobierno como el Sistema Nacional de Inversión Pública (SNIP) y el Marco 
Macroeconómico Multianual, considerar el cambio climático en los planes de Reducción de Pobreza y el Plan Perú 2021.

\section{CASO: LA CADENA PRODUCTIVAALGODÓN - TEXTIL - CONFECCIONES}

El 1\% de las empresas del sector son grandes y detentan el 94\% del valor de las exportaciones. Y si bien el sector enfrenta aún serias limitaciones, ha logrado mantenerse a flote y crecer en medio de la crisis internacional, que ha afectado de manera significativa al sub sector. Entre ellas está la falta de personal capacitado para el sector: especialistas en control de calidad, en métodos de producción y supervisores de planta. Existen pocas escuelas para mandos medios, para personal especializado en tejeduría, tintorería, acabado de telas y corte, así como es escasa la formación de operadores de máquinas de costura. Así mismo, hay poca inversión en fabricación de hilados, se estima en no menos de 10 millones.

La producción interna de textiles y confecciones contribuye aproximadamente con el 16.5\% del PBI Manufacturero (textil 9.4\% y confecciones $7.1 \%$ ) y el $2.6 \%$ del PBI global, y ha venido mostrando un ritmo acelerado de crecimiento desde el año 2000, con una pequeña desaceleración en el 2006, y una abrupta caída en el 2009.

La cadena productiva integra el conjunto de eslabones que conforma un proceso económico, desde la materia prima a la distribución de los productos terminados. Las etapas de la cadena van desde la producción de la materia prima (algodón, lana, pelos finos y fibras manufacturadas sintéticas y artificiales) hasta la confección de una gran variedad de productos. Las etapas intermedias de la cadena están reservadas para la fabricación de hilados (de algodón, de fibras manufacturadas, de lana y pelos finos), tejidos (planos y de punto) y el teñido y acabado de tejidos.

Es importante el encadenamiento hacia atrás del sector; desde la provisión de las principales materias primas e insumos, como el algodón, la lana, los pelos finos y la seda, entre otras fibras vegetales de menor importancia; así como las fibras y filamentos sintéticos provenientes de la industria petroquímica y fibras y filamentos artificiales que son obtenidos en el laboratorio. También son importantes los encadenamientos hacia adelante con la industria que le provee de servicios complementarios como los sectores plásticos, papeles y la cadena de comercialización interna y externa.

La falta de integración de esta cadena algodón-textil-confecciones, la califica como la cadena de la discordia. Si por un lado los algodoneros piden subir los aranceles para la importación de algodón, los confeccionistas piden que los rebajen, para no encarecer más sus costos. Así, la producción nacional de fibra es ya desde inicios de la última década insuficiente para abastecer la demanda de la industria textil nacional, especialistas en campo como el Dr. Juan Lazo, 
Director de Investigación del IPA señala que fácilmente este déficit alcanza el $80 \%$, lo que va contra nuestra marca reconocida mundialmente: Pima Peruano.

El Ing. Abel Basurto, Director del Proyecto Algodón de la UNALM, percibe cambios climáticos importantes en la costa central, incremento de temperatura, acompañado de mayor luminosidad y radiación, lo que está permitiendo una mayor precocidad en el cultivo. Así como nuevas oportunidades de siembra en los valles interandinos.

\section{LA ADAPTABILIDAD SE BASA EN UNA CADENA PRODUCTIVA INTEGRADA: EL ALGODÓN ORGÁNICO}

Esta cadena tiene una serie de ventajas, para los consumidores, las prendas elaboradas con algodón orgánico disminuye la posibilidad de reacciones alérgicas, así mismo contribuye con el ecosistema a través de la disminución del costo ambiental en aquellos lugares en donde se produce el algodón. Las parcelas utilizan solamente medios naturales para su manejo, y en muchos casos únicamente insumos endógenos, favoreciendo el reciclaje de diversos componentes en el ecosistema y no exponiendo a los agricultores a una intoxicación progresiva. Algunos autores aseguran además, que el algodón orgánico es mucho más suave que el algodón común, permite a la piel una mayor ventilación y respiración. En la confección de un polo o T-shirt de algodón convencional se usa más de un cuarto de kilogramo de sustancias químicas tóxicas y para un par de pantalones jeans se utilizan dos tercios de kilogramo de químicos tóxicos.

La gran ventaja para el Perú es que existe una mayor producción de fibra extra larga ELS, la cual tiene una mayor cotización y aceptación en el mercado internacional. Los otros países de la región poseen generalmente fibras de tipo medio y no cuentan con la fama que tiene el extra largo del Perú. Los primeros intentos por organizar la cadena fueron fallidos: (Biotrading y Malecón Chorrillos). Entre los proyectos consolidados se encuentran tres empresas: Tiendas Unidas S.A. (TUSA), trabajan con pequeños productores de la costa central (Pisco y Cañete), la empresa Oro Blanco, empresa de la ONG Solidaridad de Holanda, trabaja con pequeños agricultores de Cañete y Chincha, y la empresa PeruNaturtexPartners(PNP), que centra sus operaciones en Lambayeque y San Martín, y una pequeña área de Tangūis en Chincha, trabaja principalmente con asociaciones de productores. Las tres empresas son certificadas por Control Union y PNP tiene certificación fair trade de FLO (Lizárraga, 2008). Otro grupo son los proyectos instalados en la selva, representados por la empresa Romero Trading con 220 ha, y la empresa Corporación Algodonera El Pacífico S.A. El algodón es manejado por pequeños agricultores y cuentan con apoyo del Proyecto PRA5, programa de desarrollo alternativo financiado por USAID. Ambas empresas están certificadas por Control Union y la mayor parte de las áreas de estas empresas ya se encuentran con el estatus de orgánico. En 2007 
surge Bergman Rivera con algodón de color en San Martín, pero a diferencia de los otros programas no cuenta con apoyo de los proyectos especiales. Otros son New Exporten Pisco, y proyectos recientes de la ONG CIPCA en Piura con algodón Pima, y la ONG CEDEPAS en Jequetepeque.

La oferta de la fibra de algodón orgánico que se produce en el Perú está orientada hacia el mercado nacional. Esta fibra es usada por empresas locales para hilado y subproductos que si son exportados. Las empresas que se encuentran en la selva dentro del ámbito de acción de los programas de erradicación de coca, ofertan al mercado interno el algodón áspero, el cual es usado normalmente en mezcla con otras fibras en algún tipo de prendas, por ejemplo, el denim (Lizárraga, 2008).

Para mantener la integración de la cadena el precio es una variable importante, que se potencializa al sumarle otros factores importantes. En el caso del algodón Tangüis, Oro Blanco por ejemplo, paga 20\% por encima del precio de la fibra convencional, pero además asume la organización, operación y costos de la cosecha, lo cual incrementa los beneficios para el agricultor. TUSA, paga aproximadamente un $10 \%$ adicional, sobre el precio de la fibra orgánica. PNP tres a cinco puntos adicionales por la fibra orgánica, pero en este caso los agricultores tienen un sistema de asociación con el que asumen precio "fair trade" que incrementa la ganancia. Los proyectos que se encuentra implantados en la selva pagan normalmente el mismo precio que por el algodón convencional, lo cual consideramos inadecuado ya que la selva tiene un gran potencial de desarrollo a largo plazo con el cambio climático esperado.

Las empresas que en el Perú que consumen hilo orgánico son Oro Blanco y PNP (tienen sus propios programas de producción de fibra); Bergman Rivera, Hialpesa y Nettalco, que adquieren fibra de TUSA. Franky \& Ricky que adquieren hilo de Oro Blanco. Todas estas empresas también exportan el hilo a empresas de la región (p.e. Nicaragua y Brasil), Estados Unidos y países de Europa (Lizárraga, 2008). En el Perú las empresas que confeccionan algún tipo de prendas orgánicas son Peru Naturtex, Frank \& Ricky y Bergman Rivera.

El cambio climático también afecta la producción del algodón orgánico, el costo de la fertilización, con un incremento notorio del guano de islas, que se ha incrementado en los últimos años, cambios climáticos que favorezcan el incremento de insectos fitófagos y que desfavorezcan el adecuado desarrollo de la variedad seleccionada.

\section{CONCLUSIONES}

El cambio climático ya muestra sus impactos con bastante severidad en el Perú, y es que productivamente seguimos siendo dependientes de los sectores 
primarios, nuestra población no tiene el acceso a todos los servicios y es pobre en recursos. La cadena algodón textil confecciones es de larga data en nuestro país, aunque su dependencia del mercado estadounidense no le permitió su esperado desarrollo. Las recurrentes crisis han permitido que un nuevo grupo de empresarios innovadores tome la iniciativa y logre desde la ampliación de los mercados hasta la introducción de nuevos productos. Es notable también, el esfuerzo de varias comunidades especialmente costeras, las cuales en su afán de enfrentar su pobreza han iniciado programas de recuperación y producción de algodón orgánico y nativo, logrando estructurar la gobernanza de cadena ATC que el algodón convencional no ha logrado, encontrando la respuesta en empresarios innovadores, así como en sus gobiernos regionales y las autoridades del sector público de su región. Una cadena productiva integrada es la mejor respuesta ante la variabilidad climática.

\section{BIBLIOGRAFÍA}

1. Brooks, N. y Adger N. "Risk level indicators" Tyndall Centre, Reino Unido, 2003.

2. Geng Torres, Luis "El Futuro del Perú ante el cambio climático" CONAM 2006.

3. IPCC (OMM) “Cambio Climático 2007: Informe de Síntesis, Ginebra”, Suiza, 2007

4. IPCC "Cuarto informe de evaluación del IPCC: Cambio Climático IPCC", Ginebra, 2008

5. Lizárraga Travaglini, Alfonso "Estudio de mercado para algodón blanco y de color" Cordaid-Cedepas, 2008

6. MINAM "El Perú y el Cambio Climático Segunda Comunicación Nacional del Perú a la Convención Marco de las Naciones Unidas sobre Cambio Climático", 2010

7. PROCLIM "Evaluación de la vulnerabilidad física natural futura y medidas de adaptación en áreas de interés en la cuenca del río Piura”, 2004

8. Vargas, Paola "El cambio climático y sus efectos en el Perú" Serie de documentos de trabajo No 2009-14, BCRP 2009

9. Vreeland Jr., James "Recuperando el algodón nativo: una tecnología nativa para la agricultura del desierto peruano" en www.sepiaperu.com

Mi agradecimiento a mis alumnos, Gianmarco Angulo Cairo, alumno del curso Economía de los Recursos Naturales y Medio Ambiente 2013-2, y Mayra Silva Villaizán alumna del curso Economía Global 2014-1, por colaborar en la elaboración de este artículo. 